\title{
Integrated Optical Beam Steerers
}

\author{
Karel Van Acoleyen, Katarzyna Komorowska, Wim Bogaerts and Roel Baets \\ Photonics Research Group, Department of Information Technology (INTEC), \\ Ghent University - imec, Sint-Pietersnieuwstraat 41, B-9000, Belgium \\ Center for Nano- and Biophotonics (NB-Photonics), Ghent University, 9000 Ghent, Belgium \\ karel.vanacoleyen@intec.ugent.be
}

\begin{abstract}
Beam steering plays an important role in ultra-fast switching and scanning applications. This paper discusses the possibilities of the silicon photonics platform to fabricate integrated beam steerers using active phase tuning or wavelength tuning.
\end{abstract}

(C) 2012 Optical Society of America

OCIS codes: $130.3120,130.4815$.

\section{Introduction}

While fiber optic networks can provide us with Gbps connections, there is no straightforward wireless approach to transmit these large amounts of data using radio frequency (RF) techniques. Optical wireless has, however, proven to be able to provide the needed bandwidth. This paper investigates the integrated silicon photonics platform for optical beam steering applications. Apart from communication, beam steerers can also be used for interconnects [1], biological or gas sensing or LiDAR (Light Detection and Ranging) type applications. Figure 1(a) shows a schematic view of a photonic-electronic chip using off-chip beam steering for interconnecting, sensing, scanning or data communication.

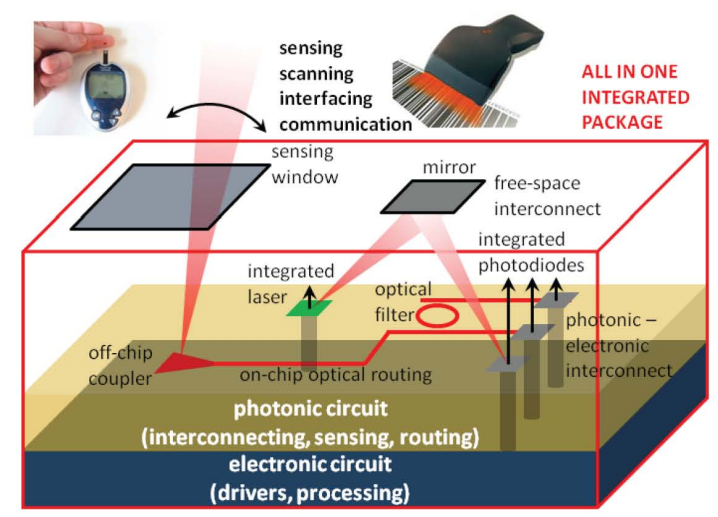

(a)

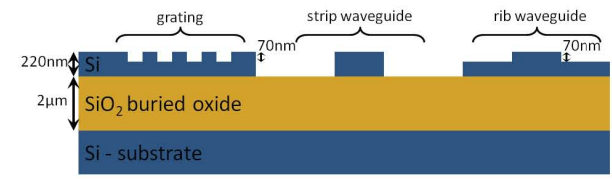

(b)

Fig. 1. (a) Schematic view of a photonic-electronic integrated chip with off-chip beam steering functionality. (b) SOI process flow: an etch of $220 \mathrm{~nm}$ and $70 \mathrm{~nm}$ allows us to fabricate a.o., gratings, strip and rib waveguides.

Next, we will discuss optical phased arrays using the silicon photonics platform. In Section 3, the different fabricated beam steerers are discussed followed by a conclusion.

\section{Optical Phased Arrays using Silicon Photonics}

An optical phased array (OPA) consists of an array of light emitting elements of which the phase can be controlled. In this way, a random phase front can be created and light can be steered non-mechanically. Liquid crystal technology is the leading OPA technology due to the advanced fabrication technology of fabricating large arrays where the phase of each pixel can be controlled. The most difficult constraint is the $\lambda / 2$ spacing to avoid higher order lobes due to sampling of the phase profile. Integrated optics can however fabricate nanometer-scale structures approaching this constraint. An in-depth review of optical beam steering techniques discussing, a.o., OPAs, can be found in [2]. 
Careful investigation of all the functionality silicon photonics has to offer shows the rationale of using this in OPA technology. Integrated lasers and detectors can act as source and detector in an OPA link. Grating couplers can act as optical antennas. Very small high-index contrast waveguides allow to route light on-chip with low loss. Phase modulation is of key importance. Several methods exist on silicon such as heating or carrier-based, making very fast phase modulation possible. Here, we have used the thermo-opic effect, having switching frequencies of several tens to hundreds of kHz.

In this paper, deep-UV optical lithography was used to fabricate the integrated beam steerers. This is a CMOS (Complementary Metal-Oxide Semiconductor) compatible platform allowing mass-fabrication. The beam steerers are processed on a $200 \mathrm{~mm}$ Silicon-On-Insulator (SOI) wafer with a $2 \mu \mathrm{m}$ buried oxide layer and a $220 \mathrm{~nm}$ top layer. Two etch steps are used in this process using deep-UV $193 \mathrm{~nm}$ lithography. The fabrication details can be found in [3]. There is one full etch of $220 \mathrm{~nm}$ that allows fabricating strip waveguides, deep etched gratings and an etch of $70 \mathrm{~nm}$ to fabricate rib waveguides, taper sections and gratings as can be seen schematically in Figure 1(b). These designs were part of MPW (Multi Project Wafer) shuttle runs organized by ePIXfab [4].

\section{Integrated Beam Steerers}

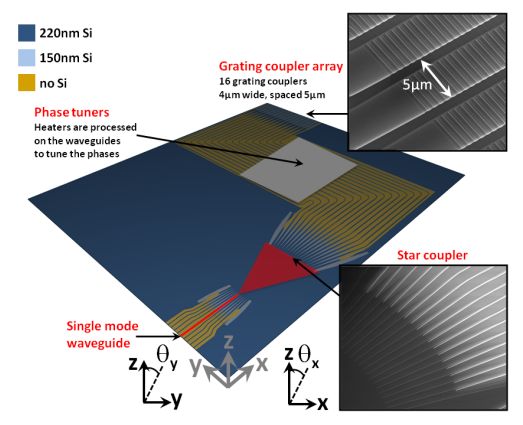

(a)



(b)

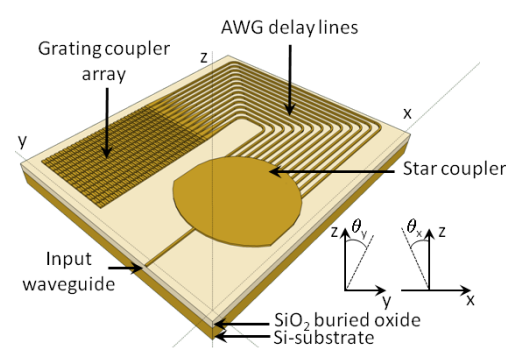

(c)

Fig. 2. (a) One-dimensional OPA with 16 addressing electrodes: the heaters can be tuned to impose a random phase front. (b) Schematic view of a two-dimensional OPA on SOI. (c) Two-dimensional dispersive beam scanner on SOI.

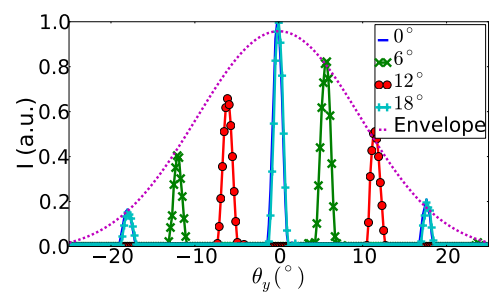

(a)

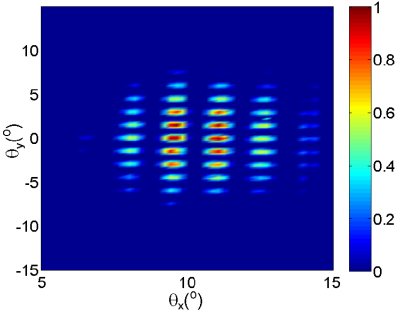

(b)

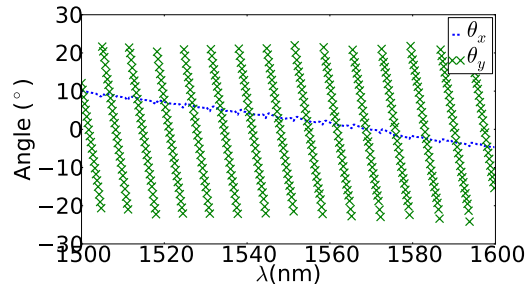

(c)

Fig. 3. (a) Measured far-field in the $\theta_{y}$-direction of a 1D OPA using thermo-optic steering at $1550 \mathrm{~nm}$. The dashed line shows the envelope of the far-field pattern. (b) Measured far-field pattern of a $2 \times 2 \mathrm{OPA}$ at a wavelength of $1550 \mathrm{~nm}$. (c) $\theta_{x}$ and $\theta_{y}$ position of a wavelength beam steerer with an AWG of order $q=-150$ as a function of wavelength.

Figure 2(a) shows the schematic of a one-dimensional OPA with multiple electrodes. Light entering through the input waveguide is split into sixteen waveguides, each with an individual heater. At the grating coupler array, the waveguides taper to a $4 \mu \mathrm{m}$ wide waveguide, spaced $\Lambda_{y}=5 \mu \mathrm{m}$, on which a grating is etched with a $630 \mathrm{~nm}$ period and fill factor of 0.5 . These gratings will couple the light off-chip. As the gratings are diffractive elements, the $\theta_{x}$-angle will be wavelength dependent. The FWHM beam width is measured to be $2.8^{\circ}$ at $1550 \mathrm{~nm}$ and the beam is steered over 
a $8.0^{\circ}$ range for a $60 \mathrm{~nm}$ wavelength shift. The out-coupling efficiency of the grating is around $38 \%$. In Figure $3(\mathrm{a})$, a cross-sectional view of the far-field in the $\theta_{y}$-direction is shown when the beam is steered at different angles using the thermo-optic phase tuners. The sidelobes (spaced $\arcsin \left(\lambda / \Lambda_{y}\right)=18^{\circ}$ ) are visible due to the large spacing of $5 \mu \mathrm{m}$. When steering at an $\theta_{y}$-angle of $18^{\circ}$ (being the free-spectral range of the array), the far-field coincides with the original far-field at $0^{\circ}$. More information can be found in [5] and other interesting work in [6]. These structures can also be used for efficient light collection of scattered light as discussed in [7].

A two-dimensional beam steerer has been fabricated in [8] consisting of a two-dimensional array of focusing grating couplers as shown in Figure 2(b). Due to the necessary routing, the element spacing is large and the fill factor is low, resulting in multiple higher-order grating lobes as seen in Figure 3(b).

Beam steering can also be done by wavelength tuning using diffractive elements. The steering principle is based on scanning a beam slowly in one direction while it is steered very fast in the other direction using a low (outcoupling grating) and high order (Arrayed Waveguide Grating, AWG) grating, respectively. The two-dimensional dispersive beam scanner is shown schematically in Figure 2(c). Light coming from the input waveguide is split into 16 waveguides, $2 \mu \mathrm{m}$ spaced. There is a fixed delay length $\Delta L_{y}$ between each waveguide, which forms the AWG. The end of each waveguide tapers to an $800 \mathrm{~nm}$ width on which a grating is etched. The order $q$ of the AWG determines the steering speed in the $\theta_{y}$-direction. The position of the beam as a function of wavelength for one of the fabricated components with an AWG of order $q=-150$ can be found in Figure 3(c). The $\theta_{x}$-angle varies slowly while the $\theta_{y}$-angle varies quickly when changing the wavelength. A total coverage range of $15^{\circ} \times 50^{\circ}$ is obtained for a $100 \mathrm{~nm}$ wavelength shift. The steering speed $\mathrm{d} \theta_{x, y} / \mathrm{d} \lambda$ is measured to be $0.148^{\circ} / \mathrm{nm}$ and $6.5^{\circ} / \mathrm{nm}$ in the $\theta_{x^{-}}$and $\theta_{y}$-direction, respectively. More information can be found in [9].

\section{Conclusion}

Beam steering was investigated in this paper using a phased array approach. Therefore, we have investigated the possibilities of the silicon photonics platform to fabricate these beam steering elements. This platform allows the fabrication of very small structures and compact phase modulators bringing the key ingredients to fabricate an OPA together in an integrated platform. Several designs such as one-dimensional OPAs with active phase tuning and wavelength tuning possibilities have been shown, clearly showing the potential of the silicon photonics platform in beam steering applications.

\section{References}

1. D. A. B. Miller, "Device Requirements for Optical Interconnects to Silicon Chips," Proceedings of the IEEE 97, 1166-1185 (2009).

2. P. F. McManamon, P. J. Bos, M. J. Escuti, J. Heikenfeld, S. Serati, H. K. Xie, and E. A. Watson, "A Review of Phased Array Steering for Narrow-Band Electrooptical Systems," Proceedings of the IEEE 97, 1078-1096 (2009).

3. S. K. Selvaraja, P. Jaenen, W. Bogaerts, D. Van Thourhout, P. Dumon, and R. Baets, "Fabrication of Photonic Wire and Crystal Circuits in Silicon-on-Insulator Using 193-nm Optical Lithography," Journal of Lightwave Technology 27, 4076-4083 (2009).

4. ePIXfab, "The silicon photonics platform," http://www.epixfab.eu/ (2011).

5. K. Van Acoleyen, K. Komorowska, W. Bogaerts, and R. Baets, "One-Dimensional Off-Chip Beam Steering and Shaping Using Optical Phased Arrays on Silicon-on-Insulators," IEEE Journal of Lightwave Technology 29, 3500-3505 (2011).

6. J. K. Doylend, M. J. R. Heck, J. T. Bovington, J. D. Peters, L. A. Coldren, and J. E. Bowers, "Two-dimensional free-space beam steering with an optical phased array on silicon-on-insulator," Optics Express 19, 21,59521,604 (2011).

7. K. Van Acoleyen, E. M. P. Ryckeboer, W. Bogaerts, and R. Baets, "Efficient Light Collection and DirectionOf-Arrival Estimation using a Photonic Integrated Circuit," IEEE Photonics Technology Letters 24, 933-935 (2012).

8. K. Van Acoleyen, H. Rogier, and R. Baets, "Two-dimensional optical phased array antenna on silicon-oninsulator," Optics Express 18, 13,655-13,660 (2010).

9. K. Van Acoleyen, W. Bogaerts, and R. Baets, "Two-dimensional Dispersive Off-chip Beam Scanner Fabricated on Silicon-On-Insulator," IEEE Photonics Technology Letters 23, 1270-1272 (2011). 